\title{
Carrières amoureuses et réinventions romantiques
}

\section{Cécile Piret ${ }^{1}$}

[Résumé] L'amour romantique aurait subi ces dernières années d'importants désenchantements face à des sphères d'évaluation des relations amoureuses plus rationalisantes. Tiré d'une enquête réalisée à Bruxelles avec des jeunes hétérosexuels (25-35 ans) issus des classes moyennes, cet article analyse des carrières amoureuses empilant différents modèles amoureux. L'étude montre que ces jeunes œuvrent à un processus d'ajustement de leurs croyances romantiques pour rendre possible des formes souhaitées d'engagement.

Mots-clés : amour romantique, carrière amoureuse, croyance, hétérosexualité.

[Abstract] In the recent years, romantic love would have suffered from disenchantment with the rise of the rationalisation of love relationships. This article is based on a research conducted amongst young heterosexual people from middle-class backgrounds, aged between 25 and 35 and living in Brussels. It analyses how love careers deal with different love models. The study shows how young people work to adjust their romantic beliefs to enable desired forms of commitments.

Keywords: romantic love, love career, belief, heterosexuality.

\section{Introduction}

On observe ces dernières décennies une évolution des modalités du discours sur l'amour et sur les formes de conjugalité dans la société occidentale contemporaine. Dans la continuité des travaux d'Eva Illouz (1997; 1998 ; 2012 ; 2013), cet article se propose d'interroger la prégnance et les formes de réinvention du modèle culturel de l'amour romantique chez des jeunes hétérosexuels issus de la région bruxelloise ${ }^{2}$. Cette classe d'âge (25-35 ans) est en effet particulièrement liée à la projection de soi dans des relations amoureuses qui se concrétisent en projets conjugaux. L'expérience amoureuse se heurte, de fait, très souvent au choix de l'engagement, et accompagne un travail de réflexivité propre à faire émerger des sphères d'évaluation, c'est-à-dire des ensembles de principes et de valeurs auxquels les acteurs font référence pour évaluer leurs relations amoureuses (Boltanski, Thévenot, 1991). Dans ce cadre, l'amour romantique serait de plus en plus mis en doute par des acteurs séduits par des évalua-

1 F.R.S-FNRS, Université libre de Bruxelles, METICES.

2 L'enquête a été réalisée entre 2012 et 2013 dans le cadre d'un mémoire de master en sociologie. Voir encadré méthodologique. 
tions rationalisantes des expériences amoureuses, venant de l'évolution des rapports sociaux, notamment de genre. Cependant, au vu de l'omniprésence des représentations romantiques dans les flux culturels, nous pensons qu'elles continuent à fonctionner comme socialisations importantes.

Notre étude présuppose que l'expérience amoureuse est configurée par des structures socio-économiques et culturelles dont les effets traversent la sphère privée, sans que l'on puisse a priori définir la nature de ceux-ci. Nous présenterons des éléments de ces structures qui nous semblent fondamentaux. Ensuite, parce que cette étude s'est avant tout intéressée au sujet amoureux, nous proposerons d'analyser l'amour en tant qu'apprentissage et croyance, configurant les pratiques et les émotions, et ce, par le concept de « carrière amoureuse ».

\section{Sociologiser le sujet amoureux}

L'importance du sentiment amoureux n'a cessé de croître dans la société contemporaine. Les institutions du mariage et de la famille ont, durant le XVIII et le XIX ${ }^{e}$ siècle, progressivement été soumises à ce nouvel ordre de l'authenticité des émotions, pour en devenir la norme dès le $\mathrm{XX}^{\mathrm{e}}$ siècle. Les fonctions lignagères du mariage, auparavant centrales, et bien qu'elles restent importantes, laissent place au sentiment amoureux comme principe de légitimation de l'union (Shorter, 1977; Ferry, 2012). Cette tendance s'accompagne de la normalisation d'un modèle familial dominant, dont la caractéristique principale est l'intrusion des sentiments dans trois domaines : dans le choix du conjoint, dans les relations parentales, et dans la valorisation des liens internes à la famille nucléaire au détriment de liens avec les communautés environnantes (Zelizer, 1994 ; Shorter, 1997; de Singly, 2004).

L'amour romantique va ainsi consacrer le lien entre sentiment amoureux et une forme de conjugalité, à travers les normes, les valeurs et les idéaux qu'il véhicule. Selon Chaumier (2004), nous pouvons retenir trois éléments idéal-typiques de ce lien: un désir d'amour pérenne, puisque «la relation romantique veut se persuader qu'elle apportera forcément le bonheur, le confort et la sécurité et qu'elle durera toujours » (Chaumier, 2004, 278) ; une exclusivité affective et sexuelle ; et des aspirations familialistes, tendant vers le mariage et la descendance.

Dans sa thèse sur l'amour romantique dans la société capitaliste avancée, Eva Illouz (1997) introduit une contradiction dans la manière dont celui-ci est vécu. L'auteure constate que le romantisme et le couple ont progressivement été l'objet de films, publicités, chansons, magazine, etc., liés au développement du marché du loisir. Si l'amour romantique reste garant du mariage, il est de plus en plus un lieu investi par des images de bonheur, de l'intensité des émotions et des « aventures» romantiques, de jeunesse, de glamour. Bref, les scénarios romantiques impliquent dorénavant la poursuite d'expériences amoureuses répétées et jouissives, la recherche de plaisir 
permanent plus que d'union éternelle. Les sujets amoureux seraient ainsi écartelés entre des aspirations « traditionnelles » de l'amour romantique dans sa dimension réaliste, et des désirs de consumérisme amoureux ${ }^{3}$, dans sa dimension hédoniste.

Parallèlement, depuis les années 60 , des modèles familiaux se multiplient. En France, la réforme du divorce de 1975 atteste la reconnaissance juridique qu'il n'y a pas une et une seule famille légitime, et pose la question de la liberté individuelle comme consubstantielle au mariage contemporain (Théry, 1996). De plus, bien que vivre en couple marié reste la situation conjugale dominante, les divorces et les unions libres augmentent. Conséquemment, les familles recomposées ou monoparentales se sont multipliées ces trente dernières années (Prioux, 2006 ; Bodier et al., 2015). Ces transformations familiales et conjugales ont comme point commun une demande croissante d'autonomie individuelle (Beck, Beck-Gernsheim, 1995; Beck-Gernsheim 1998 ; de Singly, 2004; Beck, 2008). Cette demande est productrice de formes de conjugalités plus distanciées qui ont notamment été conceptualisées par l'amour liquide (Bauman, 2004), la relation pure (Giddens, 2006) ou l'amour fissionnel (Chaumier, 2004). Bien qu'ayant leur spécificité, ces modèles conjugaux ont comme caractéristiques communes une demande croissante d'autonomie et d'indépendance des deux partenaires, une plus grande fluidité des échanges qui s'oppose au «seul et unique », une relation auto-référentielle née d'une rencontre d'intérêts selon les choix rationnels des deux partenaires ${ }^{4}$. Selon ces modèles, le couple formant une unité, sociale, affective et économique n'est plus désirable, notamment parce que l'émancipation des femmes a rendu l'idéal de fusion impossible voire inacceptable.

Si Illouz rejoint ce constat, notamment en montrant que des sphères d'évaluation venues de la technologie, de la science et de la politique ont contribué à désenchanter l'amour romantique, l'auteure admet également la coexistence de ces différents modèles. C'est en prenant pour point de départ le fait que l'amour romantique reste structurant en tant que modèle culturel que nous souhaitons voir comment il s'exprime et se réinvente face à d'autres principes d'évaluation. Particulièrement, nous regardons la manière dont le romantisme est traversé par deux tensions : la tension entre hédonisme et réalisme, et la tension entre fusion et autonomie.

À échelle individuelle, nous interrogeons la coexistence de ces normes en prenant comme angle d'analyse la notion de «carrière ». Développée à l'origine en sociologie du travail, Hughes (1958) la définit comme le parcours ou la progression d'une per-

3 Nous entendons par "consumérisme amoureux» la valorisation par les individus de relations amoureuses qu'ils peuvent prendre et jeter facilement, et pour lesquelles le rapport à l'autre se réalise uniquement dans une optique sécuritaire, en minimisant le plus possible les formes d'attachements. De plus, les individus concernés par ce consumérisme s'inscrivent dans des relations en série dans un objectif d'accumulation.

4 La relation auto-référentielle signifie une relation évaluée pour elle-même, pour les satisfactions qui en sont retirées et qui sont régulièrement réévaluées par le couple. II n'existe pas d'autre principe supérieur au nom duquel la relation devrait durer. 
sonne au cours de sa vie professionnelle, prenant place dans un espace-temps particulier. L'importance de la temporalité permet d'envisager la co-construction entre le parcours individuel et les changements en cours dans son environnement social. Becker (1963), dans son étude sur les carrières déviantes, est le premier à sortir la notion du cadre strict de la sociologie du travail. Nous l'utilisons ici afin de comprendre comment un certain rapport à l'amour se construit, par succession d'expériences et d'apprentissages, sans réduire la notion à ceux-ci. La notion de " carrière amoureuse " permet en effet de rendre compte de manière processuelle d'une vie amoureuse inscrite dans des mondes amoureux normés. Elle progresse dans une certaine culture affective. Les émotions, comme les comportements, sont soumis à des impératifs sociaux et à des normes relationnelles (Mauss, 1921; Le Breton, 2004). Les individus sont socialisés par un contrôle social des émotions, et ils apprennent à produire un travail émotionnel visant à supprimer ou à susciter les émotions requises (Fernandez et al. 2008). La carrière amoureuse envisage également les changements sur un fond de continuité. Elle ne s'arrête pas après chaque relation, mais se construit sur des discontinuités, sur la répétition d'attachements et de détachements. La rupture amoureuse, comme nous le verrons, est importante dans ce processus. Elle peut, à certains égards, être vue comme un point de bifurcation (Voetgli, 2004), c'est-à-dire un moment important de redéfinition de l'identité sociale, mais aussi d'un rapport à l'amour. Enfin, le concept de «carrière » permet une analyse compréhensive et une objectivation des trajectoires individuelles (Darmon, 2008). Facilitant l'analyse comparative entre différents récits de vie amoureuse, elle permet d'accéder, à partir d'observations phénoménologiques, à des mondes de croyances plus larges.

\section{Encadré méthodologique}

L'enquête a été réalisée par entretiens semi-directifs avec quinze jeunes hétérosexuels ${ }^{5}$ de 25 à 35 ans, 9 femmes et 6 hommes, issus de la région bruxelloise. Nous avons choisi d'interroger des jeunes issus des classes moyennes et des classes moyennes supérieures, en partant de l'hypothèse, formulée ailleurs (Illouz, 2012 ; Giddens, 2006), que ce sont les milieux sociaux les plus disposés à adopter les référentiels de l'individualisme et de l'autonomie ${ }^{6}$. Nous avons retenu comme indicateur le niveau de diplôme: tous les jeunes interrogés avaient un diplôme d'études

5 C'est un parti pris de l'enquête de n'avoir interrogé que des personnes hétérosexuelles. Si des représentations romantiques configurent également les relations homosexuelles, elles ne conduisent pas forcément aux mêmes pratiques et, notamment n'ont pas le même effet hétéro-normatif. Pour cette raison, nous ne pouvons pas généraliser nos données d'enquête en dehors des frontières de l'hétérosexualité.

6 II serait cependant intéressant de pouvoir comparer les expériences en fonction du milieu social. Notamment, Clair (2011) montre que l'éthos égalitaire se retrouve dans la jeunesse populaire rurale et de cité, mais sous des modalités spécifiques à ce milieu. 
supérieures, universitaire ou non, ou étaient en train de le réaliser. Il s'agit également de jeunes qui ont les ressources nécessaires pour pouvoir prendre leur indépendance économique. Le corpus d'informateurs a aussi été construit dans l'optique de privilégier des personnes vivant des situations amoureuses variées. Dans une perspective genrée, nous avons cherché tant les similitudes que les différences entre hommes et femmes. L'enquête a porté sur les représentations liées à l'amour ${ }^{7}$ et sur les récits de leur propre vie amoureuse. De manière similaire à la méthode des récits de vie (Bertaux, 2013) qui étudie différentes dimensions du parcours biographique avec des séquençages temporels, nous avons articulé la vie amoureuse et les temps structurant le parcours de ces jeunes (les études, l'arrivée sur le marché du travail, le premier emploi, etc.). L'enquête $a$, de ce fait, une dimension narrative très importante qui, bien qu'elle soit éloignée des pratiques réelles, a l'avantage de faire émerger des systèmes de représentations liés au modèle culturel du romantisme.

\section{Déclinaisons romantiques}

\subsection{Le maintien d'une définition idéalisée de l'amour}

Alors que le sentiment amoureux se vit de manière si intime et singulière pour l'individu, l'emprise de logiques sociales sous-tendant la trame des expériences amoureuses et de leur mise en récit laissent percevoir un nombre limité de répertoires d'interprétations (Pagès, 2008). Ainsi, lorsqu'il a été demandé aux enquêtés une définition de l'amour, ancrée dans leurs vécus, une caractéristique majeure a été mise en avant: l'enchantement. Tout se passe comme si les acteurs s'assuraient d'interpréter leurs relations amoureuses hors de toute forme de raison:

C'est souvent un peu inexplicable, c'est comme ça qu'on sait qu'on est amoureux.

Si on cherche des raisons, on peut en trouver bien sûr mais il y a une part de mys-

tère, il y a quelque chose qui nous échappe. Vraiment, profondément, la raison nous échappe. Je préfère que ça reste mystérieux. ( $\operatorname{Marc}^{8}, 35$ ans, célibataire ${ }^{9}$ )

L'amour est vu comme résultant d'un hasard ou d'une préférence irrationnelle. Il est assez caractéristique que les acteurs se réfèrent à une économie du don comme mode régulateur des échanges conjugaux. Au nom de l'amour, les individus sont disposés à

7 Ces représentations ont été interrogées par deux types de question : la première était de demander aux enquêtés leur propre définition de l'amour. La deuxième demandait aux enquêtés s'ils avaient été touchés/avaient apprécié certains produits culturels (chanson, film, livre) abordant le thème de l'amour et d'expliciter quelles en étaient les raisons.

8 Les prénoms des enquêtés ont été changés.

9 Les catégories concernant les «statuts amoureux » sont propres aux enquêtés. Dans cette étude, le terme de « célibat » ne réfère pas à la situation de ne pas être marié(e), mais bien à celle de ne pas être engagé dans une relation amoureuse réciproque. 
donner d'eux-mêmes, à consacrer du temps et de l'énergie à l'autre, sans exprimer la nécessité d'un contre-don (Mauss, 2007). Sous des formes plus intenses ou passionnelles, la relation de don a parfois été décrite sous le référentiel du sacrifice pour l'autre.

Il est intéressant de saisir le décalage entre ces définitions très idéalisées de l'amour et des réalités sociales qui, même esquissées brièvement ici, vont à l'opposé de «l'irraison amoureuse » prônée. Les tendances homogamiques dans le choix du conjoint ont été largement documentées (Girard, 2012 ; Bozon, Héran, 2006). L'économie du don, qui peut régir dans un premier temps l'organisation de la vie à deux lors d'une mise en ménage, se transforme rapidement en économie de la dette (Kaufmann, 1992) sur fond d'une répartition des tâches ménagères toujours très inégales, y compris chez les jeunes couples (Chabaud et al., 1985; Brousse, 2000 ; Pfefferkorn, 2010). Si les dissonances entre l'idéalisation de l'amour et les pratiques sont si fortes, cela mène à nous interroger sur le « désir de mystère sur le mystère du désir» (Schurmans, Domincé, 1997 : 37).

\subsection{Le socle socialisateur romantique}

Les récits de vie amoureuse indiquent l'existence d'un point de référence sur lequel se déploient des variations durant la carrière amoureuse : celui d'un socle socialisateur romantique (Chaumier, 2004). Une première socialisation romantique, qui semble surtout féminine, consiste à vivre et à relater les premières expériences amoureuses sur le ton de la fusion et de la passion, parfois destructrices.

On était comme les deux doigts de la main, le monde n'existait plus autour de nous, c'était très très fort, tout de suite, mais aussi dans les extrêmes. Donc on était aussi à se chamailler, à se gueuler dessus et puis à s'aimer comme pas possible. Lui, il était hyper jaloux, ce que j'aimais vraiment entretenir, même si c'est un peu con de ma part. [...] On avait 19 ans, on était jeune, mais on parlait bébés, mariage: pour moi c'était l'homme de ma vie, et ça l'a été pendant un an et demi... (Amélia, 25 ans, en relation instable)

En début de carrière amoureuse, chaque relation passionnelle est vécue comme la relation, et la jalousie constitue un signe d'amour. La manière dont les individus se laissent « être emballés » par leur relation est révélatrice d'une caractéristique du romantisme hédoniste : l'amour fusionnel est vécu à travers un ensemble de rituels et de symboles qui a le pouvoir de séparer pour un temps les individus de leur environnement - de le transcender - afin d'intensifier son expérience et d'échapper momentanément à la routine du quotidien. L'amour romantique peut être associé à la catégorie " amour de jeunesse » afin d'ordonner et de justifier des dispositions amoureuses " débordantes ». Cette expérience de l'amour passion est aussi évoquée pour décrire les modalités de l'amour naissant au début de toute relation (Alberoni, 1993). Mais ces premières relations fusionnelles sont également accompagnées de projections sans condition dans l'avenir. Les relations qualifiées « d'importantes » par les enquêtés sont celles considérées comme " sérieuses », selon des critères de durée et d'investissement 
affectif : l'amour est vite considéré comme s'inscrivant dans une relation exclusive et à long terme.

Le socle socialisateur romantique semble ainsi orienter la carrière amoureuse vers la recherche d'engagements et, avec comme but ultime, la rencontre authentique (" c'est vraiment le bon ») qui permettra d'accomplir les aspirations conjugales. Les individus cherchent la constitution d'une réalité amoureuse, valorisant fortement la projection de soi-même comme étant en couple. La majorité des personnes interrogées ont des projets familiaux ou se projettent dedans :

Pour moi, François, c'est ma famille, et c'est ça l'amour. C'est un foyer, une famille, même si ce n'est qu'une seule personne. Maintenant je suis enceinte et on va être trois, mais jusque-là, François c'était tout, c'était ma famille [...] Quand je dis « un tout », je veux dire que c'est une référence, quelqu'un à qui on se réfère à tout point de vue, parce qu'on a confiance. (Éléonore, 31 ans, en couple depuis dix ans)

L'être aimé est entouré d'images familialistes, et la stabilité procurée par l'institutionnalisation du couple devient une référence presque ontologique. Les individus veulent faire de l'électivité, caractéristique majeure des choix amoureux, des relations aussi solides que les liens de parenté (Bauman, 2004). L'authenticité des émotions, le principe de confiance réciproque, mais aussi la sexualité exclusive en sont des éléments constitutifs. La touche d'absolu et de sacré qui accompagne la définition de la relation amoureuse semble porter sur la question de la fidélité.

Si Margaux avait une relation avec quelqu'un d'autre, je le vivrais comme un truc hyper dur et comme une trahison, c'est clair [...] C'est vrai que nous on construit ça d'une manière traditionnelle, c'est-à-dire qu'on vit l'un pour l'autre et pas forcément en allant voir ailleurs ou en bâtissant des relations avec d'autres. (Julien, 34 ans, en couple depuis quatre ans)

Parce que la sphère sexuelle et la sphère amoureuse restent intimement liées, une infidélité sexuelle est très souvent vécue comme une trahison amoureuse (Hippert, 2012). La fidélité n'est pas conçue uniquement comme portant sur la sexualité mais est aussi garante de la fusion affective et de la véridicité des sentiments amoureux.

\subsection{L'empilement de sphères d'évaluation: entre tensions et travail de mise en cohérence}

Les représentations romantiques ne sont cependant pas hégémoniques. Elles évoluent, durant la carrière amoureuse, à la fois par l'accumulation d'expériences individuelles et par des formes d'ajustement aux réalités amoureuses ambiantes. Selon des statistiques françaises, à quarante ans, une personne sur cinq a eu au moins trois relations importantes (Rault, Régnier-Loilier, 2015) et la proportion d'unions rompues dans les cinq premières années a augmenté fortement, tant pour les cohabitants que les couples mariés (Prioux, 2006). Dans ce contexte, les aspirations romantiques sont contrebalancées par la rhétorique du risque et par les besoins d'autonomie. C'est surtout lors de l'expression de désir de formes d'engagements, matériels et familiaux, que ceux-ci sont utilisés : 
Des enfants, c'est quelque chose que j'aimerais. J'étais déjà prêt quand j'étais avec Christelle, c'est quelque chose que j'aurais fait assez vite. Après ça fait peur, justement, d'avoir eu cette claque avec ma relation précédente. Je suis un peu moins rassuré, je me livre moins facilement, les relations peut-être en pâtissent aussi. (Nathanaël, 27 ans, célibataire depuis une semaine)

La carrière amoureuse est fondamentalement marquée par des séries de relations intimes. Chaque relation passée a socialisé l'individu à l'amour, et des expériences amoureuses passées s'actualisent dans les nouvelles. Lorsque des souffrances ont été expérimentées, elles viennent freiner les processus d'engagement pourtant souhaités par l'individu dans sa nouvelle relation. Ceux-ci sont alors traversés par la rhétorique du risque (Beck, 2008) : risque face aux attachements matériels, pour lesquels l'achat immobilier commun fonctionne comme un pari sur l'avenir ; risque face à des engagements familiaux qui nécessitent une confiance mutuelle. Si toute relation exige la construction d'une réalité sociale commune (Berger, Kellner, 2012), il y a également la perception d'un risque de se perdre dans une définition de soi exclusivement tournée vers la réalité conjugale. Chez les femmes interrogées, particulièrement, l'investissement de soi dans le couple est valorisé de manière ambivalente, entre désir de fusion et revendication d'indépendance. Il existe une tension entre des dispositions contradictoires, celles de valoriser une plus grande autonomie mais de continuer à se définir comme étant pour les autres, dans des relations de soin et de sollicitude (Löwy, 2005). Ces jeunes femmes ont exprimé à maints égards cette tension :

[...] mon premier sentiment par rapport à ce livre ${ }^{10}$, c'est quelle belle histoire d'amour! C'est magnifique d'avoir quasiment sacrifié sa vie pour son amant, parce qu'à un moment elle sort du monde, elle tombe enceinte et comme c'est un enfant illégitime, elle est condamnée à vivre dans une petite baraque et à laisser sa vie mondaine [...] d'abord je me suis dit que c'était magnifique. Et puis ma sœur s'est mise avec un gars que je n'approuve pas [...] ; j'ai vu ma sœur s'abandonner complètement, un peu comme Héloïse. Là, j'ai un peu changé de point de vue par rapport à ce livre et je me suis dit que non, ce n'est pas une bonne chose de s'abandonner comme ça de façon irraisonnée, les yeux fermés comme ça à son amour. Je préfère qu'il y ait un minimum de raison à l'amour, que ce [ne] soit pas uniquement une passion inexplicable, irrationnelle, démesurée. (Véronique, 31 ans, en couple depuis un an et demi)

Les femmes mobilisent le référentiel de l'autonomie dans une optique égalitariste. Elles expriment de manière plus ou moins explicite une méfiance envers un rôle qu'elles ne se voient pas assumer, et qui est configuré par les représentations fusionnelles de l'amour romantique. Alors que nous voyions une certaine idéalisation d'un amour aveugle dans la manière dont les enquêtés définissent l'amour, l'évaluation devient plus nuancée lorsqu'elle résulte d'une expérience douloureuse passée. Les hommes ont également exprimé des besoins d'autonomie, qui se sont particulière-

10 Véronique fait référence aux Lettres d'Abélard et d'Héloïse, écrites par Pierre Abélard (1079-1142), qui consacrent le modèle de l'amour courtois entre deux amants clandestins. 
ment reflétés dans la peur de l'engagement. Ils expriment soit une certaine valorisation de la figure du célibataire, soit un désir de cumuler les expériences avant la rencontre, in fine, de l'être authentique. Cette peur de l'engagement est caractérisée par la difficulté de se fixer sur une personne parce que l'imaginaire amoureux contemporain et occidental offre l'idée qu'il existe constamment de multiples possibilités (Illouz, 2012). Cependant, cette peur est systématiquement contrebalancée par l'envie de ne pas assumer une paternité trop tardive ${ }^{11}$. Elle est ainsi propice à susciter des contradictions importantes, et, parfois, des souffrances, lorsque, par convergence de dimensions à la fois individuelles et amoureuses, les aspirations à la descendance sont saillantes. Un dernier élément, dominant parmi les enquêtés, nous semble caractéristique de l'empilement de ces normes : l'ambivalence sur le désir d'amour pérenne.

Pour moi, Mélanie, c'est la femme de ma vie, clairement, c'est ce que je ressens maintenant. Je suis bien avec elle [...], elle m'apporte beaucoup. Mais effectivement dans 20 ans, qui peut savoir ce que seront les choses, malgré toute l'envie du monde que j'ai de passer ma vie avec elle? C'est impossible de dire si dans 20 ans je serai encore avec elle. Je pense que personne ne peut dire ça, même si on a envie d'y croire, et qu'on va tout faire pour. (Niluge, 27 ans, en couple depuis trois ans)

Bien que les individus veulent y croire, les idéaux romantiques sont réajustés selon un principe de réalité qui crée l'ambivalence du discours ( "l'amour de ma vie même si... »), et provoque un certain cynisme, comme l'a confié un des enquêtés. Nous pensons que la rupture y joue un rôle majeur. Dans des carrières amoureuses largement définies par des "monogamies en série », les ruptures sont consubstantielles au parcours amoureux. Plus qu'une éventualité dès les commencements de l'amour (de Singly, 2011), la rupture, en tant qu'évènement et processus de désocialisation, constitue une nouvelle norme de l'horizon amoureux :

On s'est séparé deux fois. Une fois vers mes 24-25 ans, je ne savais pas ce que je voulais et du coup le couple est passé aussi dedans. C'était comme si j'étais étonnée moi-même qu'on avait tenu si longtemps et comme je n'avais pas d'expérience... je me disais oui, il parait que tout a une fin, tous les couples autour de moi finissent par se séparer donc nous de toute façon, ça va bien se finir un jour, alors à quoi bon tout

ça ! C'était la période «à quoi bon? » (Éléonore, 31 ans, en couple depuis 10 ans).

\section{La fonction de la croyance}

Les individus combinent donc cet horizon normatif amoureux avec des aspirations à projeter le couple à long terme. Paradoxalement, il semblerait que cette instabilité ambiante des relations amoureuses amène et justifie le discours romantique. Tout se

11 Contrairement aux idées reçues, l'enquête montre que les femmes ne sont pas les seules à ressentir à un moment donné le besoin pressant de maternité. Si les modalités sont différentes pour les hommes, ils ne sont pas moins touchés par ces formes de pression temporelle qui dévalorisent l'image d'un « père trop âgé » pour ses enfants. 
passe comme si les acteurs s'attachaient à y croire, même s'ils n'y croient plus entièrement et que certains codes sont rejetés de manière cynique. Le besoin de croire en l'amour romantique remplirait, dès lors, à un moment de la carrière, un «vide » à combler.

L'engagement en amour, on a l'impression que c'est quelque chose d'insurmontable parce que c'est comme si on était dans une société où on nous offrait la possibilité de croire que tout est possible [...] et on nous dit que ça s'étale aussi pour l'amour, c'est-à-dire que l'amour ne dure que trois ans [...] Alors que moi j'avais envie de combattre ça justement, et de croire que c'est possible de vivre toute sa vie avec quelqu'un. (Jean-Baptiste, 29 ans, célibataire)

L'enquête montre une prégnance d'aspirations familialistes que l'on peut qualifier de traditionnellement romantiques : un désir d'amour pérenne, une fidélité affective et sexuelle, une mise en ménage commune, le désir de progéniture. Malgré une diversification des projets conjugaux ${ }^{12}$, ceux-ci restent confinés à l'intérieur des frontières de la monogamie et de la cohabitation nucléaire, même avec un recul de l'aspiration au mariage. La famille, dès lors, reste une valeur importante. Comment l'amour et la famille peuvent-ils être si importants dans la vie des individus alors qu'ils n'ont jamais été aussi instables ? Beck et BeckGernsheim (1995) soulèvent ce paradoxe existant entre les multiples formes de désintégration de la famille classique et son idéalisation. D'un côté, la famille continue à être perçue comme lieu refuge, dans lequel est puisé des modèles idéaux de relations humaines (Chaumier, 2004; Bourdieu, 1993). La famille est investie de sacré, dans le sens de « se sacrifier pour » (Zelizer, 1994 ; Ferry, 2010). De l'autre côté, la famille fondée sur les sentiments amoureux génère des formes incertaines de lien social. La famille est fragile et instable, au moment où elle promet d'être un lieu refuge à l'insécurité. Là où les autres contacts sociaux s'affaiblissent, l'expérience amoureuse est valorisée pour l'intensité émotionnelle qu'elle procure et dont elle semble avoir le monopole. Dans ce contexte, l'engagement que présuppose la constitution d'une famille nucléaire nécessite une justification culturelle permettant de le rendre enviable. Pour cette raison, la croyance romantique est nécessaire à l'engagement relationnel (Hippert, 2012) et est réinventée à l'aune de valeurs plus émancipatrices pour l'individu.

12 Ceux-ci n'ont pas pu être interrogés dans le cadre de cette enquête. Cependant, un certain nombre d'études attestent que les formes de conjugalités se sont fortement diversifiées ces dernières décennies. Voir notamment les cinq styles conjugaux proposés par Kellerhals et al. (2004), ainsi que l'étude de de Singly (2000) sur les formes d'individualisme dans la vie commune. 


\section{Conclusion}

Notre étude a tenté de montrer comment des jeunes hétérosexuels issus des classes moyennes œuvrent à un processus d'ajustement de leurs croyances romantiques afin qu'elles puissent continuer à être structurantes pour des formes souhaitées d'engagement. Au lieu de s'en défaire, ces jeunes travaillent à réinventer un modèle romantique qui, tout au moins dans les récits, intègre des dimensions venant de sphères d'évaluations nouvelles, et qui, en tant que telles, sont plutôt opposées au romantisme. S'ils n'arrivent pas toujours à intégrer ces dimensions avec les représentations romantiques, ces jeunes ont cependant exprimé à quel point ils avaient envie de continuer à y croire. Nous n'avons donc pas retrouvé de manière "pure » des formes rationalisées du choix amoureux, tant décrites dans la littérature sur le sujet. La notion de carrière amoureuse s'est avérée utile afin de saisir comment les jeunes articulent les deux ensembles de tensions hédonisme/réalisme et fusion/autonomie qui traversent le modèle romantique. Tout le long de la carrière, le romantisme est mobilisé soit comme valeur, soit comme expérience, soit comme idéal à atteindre. Les individus y croient avec une intensité changeante, selon les étapes de leur vie amoureuse.

D'abord, les représentations idéalisées de l'amour ainsi que les premières expériences amoureuses indiquent la prégnance très forte du modèle culturel de l'amour romantique. Chez les femmes, surtout, les caractéristiques hédonistes et fusionnelles sont valorisées. Elles ne le sont cependant qu'à des moments précis de la carrière : lors des premières relations amoureuses et lors de l'amour naissant ${ }^{13}$. La catégorie " amour de jeunesse » permet de normaliser l'expérience et de la rendre désirable. Par la suite, ces expériences sont vues comme dangereuses pour la définition du soi féminin. Chez les hommes, nous retrouvons les modalités de l'hédonisme et de l'autonomie aux moments du choix d'engagement(s). L'hédonisme amoureux envisage le marché matrimonial comme une multiplication des possibles. Se fixer sur «l'un et l'unique » est perçu comme plus difficile, car ils s'avèrent être nombreux. Cependant, l'envie de paternité amène les jeunes hommes à rejoindre une définition plus réaliste du romantisme.

Ensuite, les aspirations familialistes prennent place sous une double modalité. D'une part, elles renouent avec un répertoire réaliste de l'amour romantique, qui consiste à envisager la relation amoureuse d'une manière moins passionnelle, plus stabilisée, pour laquelle il faut "faire des efforts", "tout faire pour que cela marche». D'autre part, alors que le mode d'engagement serait propice à une rationalisation du choix, c'est sous le motif de la croyance qu'il s'exprime. Il est certain que ces rationalisations existent, notamment en termes d'investissement dans l'avenir, de convergence d'intérêts et de statut social. Mais si celles-ci sont transparentes dans les maga-

13 La narration entourant l'amour naissant n'est cependant pas genrée. Cet amour est valorisé tant par les hommes que par les femmes. 
zines self-help ${ }^{14}$, elles ne sont pas forcément avouables pour les individus. L'amour romantique reste la justification dominante des engagements en tant que fiction structurante, bien qu'il soit réajusté aux réalités ambiantes des relations amoureuses contemporaines, comme l'horizon de la rupture.

\section{Bibliographie}

Alberoni F. (1993 [1979]), Le choc amoureux, l'amour à l'état naissant, Paris, Pocket.

Bauman Z. (2004), L'amour liquide. De la fragilité des liens entre les hommes, Paris, Le Rouergue.

BECK U. (2008 [1986]), La société du risque, Paris, Flammarion.

Beck U., Beck-Gernsheim E. (1995), The Normal Chaos of Love, Cambridge, Polity Press.

BeCK-GeRnSHeIm E. (1998), « On the way to a post-familial family. From a community of need to elective affinities », Theory, Culture e\& Society, vol. 15, p. 53-70.

BeCKer H. (1985), Outsiders. Études de sociologie de la déviance, Paris, A.-M. Métaillé.

Berger P., Kellner H. (2012), « Le mariage et la construction de la réalité », in P. Berger, T. Luckmann, La construction sociale de la réalité, Paris, Armand Colin, p. 293-319.

BERTAUX D. (2013 [1997]), Le récit de vie, Paris, Armand Colin.

Bodier M., Buisson G., Lapinte A., Robert-Bobee I. (2015), « Couples et familles : entre permanences et ruptures », Insee Références («Vue d'ensemble »).

BouRdieu P. (1993), "A propos de la famille comme catégorie réalisée », Actes de la recherche en sciences sociales, vol. 100, p. 32-36.

Boltanski L., TheVenot L. (1991), De la justification. Les économies de la grandeur, Paris, Gallimard.

Bozon M., Heran F. (2006), La formation du couple, Paris, La Découverte ("Grands Repères »).

BROUSSE C. (2000), «La répartition du travail domestique entre conjoints: permanences et évolution de 1986 à $1999 »$, France, Portrait social, Insee.

14 Ceci pourrait par exemple expliquer la surestimation du psychologisme et du rationalisme dans les travaux de Giddens (2006) et d'Illouz (2012). Ceux-ci ont élaboré une partie de leur modèle sur l'analyse de la littérature abondante du développement personnel. Dans le quotidien amoureux, cependant, ces modèles sont moins consistants et hégémoniques. 
Clair I. (2011), «La découverte de l'ennui conjugal», Sociétés contemporaines, vol. 3, $\mathrm{n}^{\circ} 83, \mathrm{p} .59-81$.

Chabaud-Richter D., Fougeyrollas-Schwebel F., Sonthonnax F. (1985), Espace et temps du travail domestique, Paris, Méridiens Klincksieck.

Chaumier S. (2004 [1999]), La déliaison amoureuse, de la fusion romantique au désir d'indépendance, Paris, Armand Colin.

DARMON M. (2008), « La notion de carrière : un instrument interactionniste d'objectivation », Politix, vol. 2, n 82, p. 149-167.

DE Singly F. (2000), Libres ensemble: l'individualisme dans la vie commune, Paris, Nathan.

De Singly F. (2011), Séparée, vivre l'expérience de la rupture, Paris, Armand Colin.

DE Singly F. (2004 [1993]), Sociologie de la famille contemporaine, Paris, Armand Colin.

Fernandez F., Leze S., Marche H. (Coord.) (2008), Le langage social des émotions, Paris, Economica-Anthropos.

FERRY L. (2012), La révolution de l'amour, Paris, Plon.

Giddens A. (2006), La transformation de l'intimité. Sexualité, amour et érotisme, Paris, Hachette.

Girard A. (2012 [1964]), Le choix du conjoint. Une enquête psycho-sociologique en France, Paris, Armand Colin.

HIPPERT D. (2012), «L'amour naissant : les ressorts dramaturgiques d'un élan sentimental », SociologieS («Premiers textes »), p. 2-24.

Hughes E. (1958), Men and their work, Michigan, University of Michigan Libraries.

Illouz E. (1997), Consuming the romantic utopia. Love and the cultural contradictions of capitalism, Berkeley et Los Angeles, University of California Press.

Illouz, E. (1998), «The lost innocence of love: romance as a postmodern condition », Theory, Culture, Society, vol. 15, p. 161-186.

Illouz E. (2012), Pourquoi l'amour fait mal. L'expérience amoureuse dans la modernité, Paris, Seuil.

Illouz E., CARnEVAli B., Coccia E. (2013), « La liberté organisée de l'amour. Entretien avec Eva Illouz », Diogène, vol. 1, nº 241, p. 115-120.

Kaufmann J.-C., (1992), La trame conjugale, analyse du couple par son linge, Paris, Nathan.

Kellerhals J., Widmer E., Levy R. (2004), Mesure et démesure du couple. Cohésion, crises et résilience dans la vie des couples, Paris, Payot. 
LE BRETON D. (1998), Les passions ordinaires, anthropologie des émotions, Paris, Armand Colin.

Löwy I. (2006), L'emprise du genre. Masculinité, féminité, inégalité, Paris, La Dispute (« Le genre du monde »).

Mauss M. (1921), «L'expression obligatoire des sentiments», Journal de psychologie, vol. 18, p. 425-433.

Mauss M. (2007 [1925], Essai sur le don. Forme et raison de l'échange dans les sociétés archaïques, Paris, PUF («Quadrige Grands textes »).

PAges M. (2008), L'amour et ses histoires. Une sociologie des récits de l'expérience amoureuse, Paris, L'Harmattan.

PFEFFERKoRn R. (2011), «Le partage inégal des "tâches ménagères » », Les Cahiers de Framespa, vol. 7. En ligne, consulté le 29 mars 2016. URL: http://framespa.revues.org/646.

PRIOUX F. (2006), «Vivre en couple, se marier, se séparer : contrastes européens », Populations et sociétés, Ined, $\mathrm{n}^{\circ} 422$.

RAUlt W., RegnieR-Loilier A. (2015), «La première vie en couple : évolutions récentes », Population et sociétés, Ined, $\mathrm{n}^{\circ} 521$.

SChuRmans M.-N., Dominicie L. (1997), Le coup de foudre amoureux, essai de sociologie compréhensive, Paris, PUF (« Sociologie d'aujourd'hui »).

SHORTER E. (1977), Naissance de la famille moderne, Paris, Seuil.

THÉRY I. (1996), Le Démariage. Justice et vie Privée, Paris, Odile Jacob.

Voetgli M. (2004), « Du jeu dans le Je : ruptures biographiques et travail de mise en cohérence », Lien Social et Politiques, vol. 51, p. 145-158.

ZeLIZER V. (1994), Princing the priceless child, the changing social value of children, Princeton, Princeton University Press. 